\title{
Masses of Negative Multinomial Distributions: Application to Polarimetric Image Processing
}

\author{
Philippe Bernardoff, ${ }^{1}$ Florent Chatelain, ${ }^{2}$ and Jean-Yves Tourneret ${ }^{3}$ \\ ${ }^{1}$ Université de Pau et des Pays de l'Adour, Avenue de l'Université, 64012 Pau, France \\ ${ }^{2}$ Gipsa-Lab, Département Images et Signal, 961 rue de la Houille Blanche, BP 46, 38402 Saint Martin d'Héres, France \\ ${ }^{3}$ Université de Toulouse, IRIT/ENSEEIHT/TESA, 2 rue Charles Camichel, BP 7122, 31071 Toulouse Cedex 7, France
}

Correspondence should be addressed to Florent Chatelain; florent.chatelain@gipsa-lab.inpg.fr

Received 5 June 2012; Revised 30 October 2012; Accepted 8 November 2012

Academic Editor: Edwin Ortega

Copyright (c) 2013 Philippe Bernardoff et al. This is an open access article distributed under the Creative Commons Attribution License, which permits unrestricted use, distribution, and reproduction in any medium, provided the original work is properly cited.

This paper derives new closed-form expressions for the masses of negative multinomial distributions. These masses can be maximized to determine the maximum likelihood estimator of its unknown parameters. An application to polarimetric image processing is investigated. We study the maximum likelihood estimators of the polarization degree of polarimetric images using different combinations of images.

\section{Introduction}

The univariate negative binomial distribution is uniquely defined in many statistical textbooks. However, extensions defining multivariate negative multinomial distributions (NMDs) are more controversial. Most definitions are based on the probability generating function (PGF) of these distributions. Doss [1] proposed to define the PGF of an NMD as the inverse $\lambda$ th power of a polynomial linear in each of its variables. This definition can also be found in the famous textbook [2, page 93] and the computation of its modes has been investigated in [3]. A more general class of NMDs introduced in [4] was characterized by PGFs of the form $|\mathbf{I}-\mathbf{Q}|^{\lambda}|\mathbf{I}-\mathbf{Q Z}|^{-\lambda}$, where $\lambda>0$, $\mathbf{Q}$ is an $n \times n$ matrix, and $\mathbf{Z}=\operatorname{diag}\left(z_{1}, \ldots, z_{n}\right)$. In particular, matrices $\mathbf{Q}$ yielding infinitely divisible PGFs were derived. Finally, Bar-Lev et al. [5] introduced NMDs whose PGFs are defined as the inverse $\lambda$ th power of any affine polynomial. Necessary and sufficient conditions on the coefficients of this affine polynomial were derived to obtain the PGF of a multivariate distribution defined on $\mathbb{N}_{0}^{n}$ (where $\mathbb{N}_{0}$ is the set of nonnegative integers) [6]. These very general multivariate NMDs were recently used for image processing applications in [7].
The family of NMDs introduced in [5] can be defined as follows. Let us denote $[n]=\{1, \ldots, n\}$ the set of the $n$ first nonzero integers. We denote $\mathbf{z}^{T}=\prod_{t \in T} z_{t}$ as the monomial obtained by multiplying all the entries of the vector $\mathbf{z}=\left(z_{1}, \ldots, z_{n}\right) \in \mathbb{R}^{n}$ whose indexes belong to $T$, where $T \subset[n]$ stands for any subset of the indexes. Let $P_{n}(\mathbf{z})=$ $\sum_{T \subset[n], T \neq \emptyset} p_{T} \mathbf{z}^{T}$ be an affine polynomial with respect to the $n$ variables $\left(z_{1}, \ldots, z_{n}\right)$ such that $1-P_{n}(\mathbf{1}) \neq 0$. The NMD distribution defined at pair $\left(n, P_{n}\right)$ is represented by its PFG which is given by

$$
\mathscr{G}_{\mathcal{N} M\left(n, P_{n}\right)}(\mathbf{z})=\left(1-P_{n}(\mathbf{z})\right)^{-\lambda}\left(1-P_{n}(\mathbf{1})\right)^{\lambda} .
$$

Such laws are denoted as $\mathcal{N} \mathscr{M}\left(n, P_{n}\right)$. However, as explained in [6], all couples $\left(n, P_{n}\right)$ do not provide a valid NMD. More specifically, Bernardoff has derived a finite number of conditions over $P_{n}$ such that $\left(1-P_{n}(\mathbf{z})\right)^{-\lambda}\left(1-P_{n}(\mathbf{1})\right)^{\lambda}$ is the PGF of an NMD for all positive integer $n$. The corresponding expression of the coefficient of $\mathbf{z}^{\alpha}$ in the Taylor expansion of $\left(1-P_{n}(\mathbf{z})\right)^{-\lambda}$ is given by the formula

$$
c_{\alpha}\left(\lambda, P_{n}\right)=\sum_{k \in K_{\alpha}}(\lambda)_{|k|} \frac{\mathbf{p}^{k}}{k !},
$$


where $K_{\alpha}=\left\{k: \mathscr{P}_{n} \rightarrow \mathbb{N}\right\}$ and $\mathscr{P}_{n}$ is the set of all subsets of $[n]$. However, this expression of $c_{\alpha}\left(\lambda, P_{n}\right)$ does not allow us to explicitly compute the masses of NMDs in the general case.

As a first goal of this paper, we propose a way of computing the masses of multivariate NMDs $\mathscr{N} \mathscr{M}\left(n, P_{n}\right)$ defined above. A specific attention is devoted to bivariate and trivariate cases. In particular, it allows us to retrieve the results of [7] obtained for bivariate NMDs. The second part of the paper is devoted to the application of NMDs to image processing, more specifically to polarimetric image processing $[8,9]$. Polarimetric image processing has received a considerable attention in the image processing and optical communities (see for instance [10-12] and references therein). The state of polarization of a polarimetric image is classically characterized by the degree of polarization (DoP) whose estimation is of major importance $[13,14]$. The DoP of polarimetric images can be classically estimated by using four images associated to four different polarizations [15]. However, estimating the DoP using less than four images is interesting since it allows one to reduce the acquisition time and the resulting cost of the imaging system. As a consequence, there has been recently an increasing interest in deriving estimators of the DoP based on a reduced number of polarimetric images. Depending on the intensity of the acquired images, polarimetric images are referred to as low flux or high flux images (low flux corresponding to a small intensity and high flux to a larger intensity). DoP estimation based on a single polarimetric image was considered in $[16,17]$ under high flux and low flux assumptions. DoP estimators derived from two intensity images degraded by fully developed speckle noise were studied in $[18,19]$. Finally, imaging systems using three polarimetric images were studied in [20,21], under high flux and low flux assumptions.

This paper studies the maximum likelihood estimators (MLEs) of the square DoP based on two or three polarimetric images. These estimators are computed by maximizing the masses of bivariate or trivariate NMDs derived in the first part of this work.

The paper is organized as follows. Section 2 recalls important results on NMDs. Section 3 proposes a new way of computing masses of NMDs. A particular attention is devoted to bivariate and trivariate cases. Section 4 addresses the problem of estimating the square DoP of low flux polarimetric images using the maximum likelihood (ML) principle. Different MLEs are constructed depending on the number of available polarimetric images. Simulation results are presented in Section 5.

\section{Negative Multinomial Distributions}

An $n$-variate NMD is the distribution of a random vector $\mathbf{N}=$ $\left(N_{1}, \ldots, N_{n}\right)$ taking its values in $\mathbb{N}_{0}^{n}$ whose PGF is

$$
G_{\mathbf{N}}(\mathbf{z})=\mathbb{E}\left(\prod_{k=1}^{n} z_{k}^{N_{k}}\right)=\left[P_{n}(\mathbf{z})\right]^{-\lambda}
$$

where $\mathbb{E}$ denotes the mathematical expectation, $\mathbf{z}=\left(z_{1}, \ldots\right.$, $\left.z_{n}\right), \lambda>0$, and $P_{n}(\mathbf{z})$ is an affine polynomial of order $n$. (A polynomial $P_{n}(\mathbf{z})$ with respect to $\mathbf{z}=\left(z_{1}, \ldots, z_{n}\right)$ is affine if the one variable polynomial $z_{j} \mapsto P_{n}(\mathbf{z})$ can be written as $A^{(-j)} z_{j}+B^{(-j)}$ (for any $j=1, \ldots, d$ ), where $A^{(-j)}$ and $B^{(-j)}$ are polynomials with respect to the $z_{i}$ 's with $i \neq j$.) These discrete distributions have received much interest in the literature (see for instance [2] and the references therein). Of course, the affine polynomial $P_{n}$ has to satisfy appropriate conditions to ensure that $G_{\mathrm{N}}(\mathbf{z})$ is a PGF. These conditions include the trivial equality $P_{n}(1, \ldots, 1)=1$. However, determining all pairs $\left(P_{n}, \lambda\right)$ such that $G_{\mathbf{N}}(\mathbf{z})$ is a PGF is still an open problem (see [6], for discussions related to this problem). As explained in [6], the affine polynomial $P_{n}(\mathbf{z})$ can be rewritten

$$
P_{n}(\mathbf{z})=\frac{A_{n}\left(a_{1} z_{1}, \ldots, a_{n} z_{n}\right)}{A_{n}\left(a_{1}, \ldots, a_{n}\right)},
$$

where $a_{1}, \ldots, a_{n}$ are positive numbers and $A_{n}$ is an affine polynomial such that $A_{n}(0, \ldots, 0)=1$. The Taylor expansions of $\left[A_{n}(\mathbf{z})\right]^{-\lambda}$ and $\left[P_{n}(\mathbf{z})\right]^{-\lambda}$ in the neighborhood of $(0, \ldots, 0)$ will be denoted as follows:

$$
\begin{aligned}
{\left[A_{n}(\mathbf{z})\right]^{-\lambda} } & =\sum_{\boldsymbol{\alpha} \in \mathbb{N}_{0}^{n}} c_{\boldsymbol{\alpha}}\left(\lambda, A_{n}\right) \mathbf{z}^{\boldsymbol{\alpha}}, \\
{\left[P_{n}(\mathbf{z})\right]^{-\lambda} } & =\sum_{\boldsymbol{\alpha} \in \mathbb{N}_{0}^{n}} c_{\boldsymbol{\alpha}}\left(\lambda, P_{n}\right) \mathbf{z}^{\boldsymbol{\alpha}},
\end{aligned}
$$

where $\boldsymbol{\alpha}=\left(\alpha_{1}, \ldots, \alpha_{n}\right)$ and $\mathbf{z}^{\boldsymbol{\alpha}}=\prod_{i=1}^{n} z_{i}^{\alpha_{i}}$. Equations (3) and (4) clearly show that the masses of multivariate NMDs denoted as $c_{\alpha}\left(\lambda, P_{n}\right)$ can be expressed as follows:

$$
c_{\alpha}\left(\lambda, P_{n}\right)=c_{\alpha}\left(\lambda, A_{n}\right) \frac{\prod_{i=1}^{n} a_{i}^{\alpha_{i}}}{A_{n}\left(a_{1}, \ldots, a_{n}\right)^{-\lambda}} .
$$

\section{Masses of Negative Multinomial Distributions}

In this section, we derive new expressions for the coefficients $c_{\alpha}\left(\lambda, A_{n}\right)$ that will be used to compute the masses of NMDs. The particular cases of bivariate and trivariate NMDs will play an important role for the estimation of the DoP on polarimetric images. In order to compute the $c_{\alpha}\left(\lambda, A_{n}\right)$, we derive several results summarized in this section whereas all demonstrations are reported in the appendix.

Theorem 1. Denote $\mathfrak{P}_{n}^{*}$ as the set of nonempty subsets of $[n]=$ $\{1, \ldots, n\}$. Any affine polynomial $A_{n}$ such that $A_{n}(\mathbf{0})=1$ denoted as

$$
A_{n}(\mathbf{z})=1-\sum_{T \in \mathfrak{P}_{n}^{*}} a_{T} \mathbf{z}^{T}
$$

can be expressed as follows:

$$
A_{n}(\mathbf{z})=\prod_{i \in[n]}\left(1-a_{i} z_{i}\right)-\sum_{T \in \mathfrak{P}_{n}^{*},|T| \geqslant 2} d_{T}^{n} \mathbf{z}^{T} \prod_{i \in[n] \backslash T}\left(1-a_{i} z_{i}\right),
$$


where $|T|$ is the cardinal of the set $T$. Moreover

$$
\begin{aligned}
A_{n}(\mathbf{z})= & {\left[\prod_{i \in[n]}\left(1-a_{i} z_{i}\right)\right] } \\
& \times\left(1-\sum_{T \in \mathfrak{P}_{n}^{*},|T| \geqslant 2} d_{T}^{n} \frac{\mathbf{z}^{T}}{\prod_{i \in T}\left(1-a_{i} z_{i}\right)}\right) \\
= & {\left[\prod_{i \in[n]}\left(1-a_{i} z_{i}\right)\right] } \\
& \times\left(1-Q_{n}\left(\frac{z_{1}}{1-a_{1} z_{1}}, \ldots, \frac{z_{n}}{1-a_{n} z_{n}}\right)\right),
\end{aligned}
$$

where $Q_{n}$ is the polynomial defined by $Q_{n}(\mathbf{z})=$ $\sum_{T \in \mathfrak{P}_{n}^{*},|T| \geqslant 2} d_{T}^{n} \mathbf{z}^{T}$ and $d_{T}^{n}$ is related to the $2^{|T|}-1$ variables $a_{S}, S \in \mathfrak{P}_{T}^{*}$ as follows:

$$
d_{T}^{n}=\sum_{\substack{T \in \mathfrak{P}_{n} \\|T|>1}}^{|T|} a_{T} a^{[n] \backslash T}+(|T|-1) \prod_{i \in T} a_{i}
$$

Remark 2. In the trivariate case defined by $n=3$, the polynomial $A_{3}(\mathbf{z})$ can be expressed as

$$
\begin{aligned}
A_{3}(\mathbf{z})= & \left(1-a_{1} z_{1}\right)\left(1-a_{2} z_{2}\right)\left(1-a_{3} z_{3}\right) \\
& \times\left[1-Q_{3}\left(\frac{z_{1}}{1-z_{1}}, \frac{z_{2}}{1-z_{2}}, \frac{z_{3}}{1-z_{3}}\right)\right],
\end{aligned}
$$

where the coefficients of the polynomial

$$
Q_{3}(\mathbf{z})=b_{1,2} z_{1} z_{2}+b_{1,3} z_{1} z_{3}+b_{2,3} z_{2} z_{3}+b_{1,2,3} z_{1} z_{2} z_{3}
$$

can be determined using the relations

$$
\begin{aligned}
b_{i, j} & =a_{i, j}+a_{i} a_{j}, \quad i, j \in\{1,2,3\}, i \neq j, \\
b_{1,2,3} & =a_{1,2,3}+a_{1} a_{2,3}+a_{2} a_{1,3}+a_{3} a_{1,2}+2 a_{1} a_{2} a_{3} .
\end{aligned}
$$

The next theorem provides a relation between the coefficients of the polynomials $A_{n}(\mathbf{z})$ and $Q_{n}(\mathbf{z})$ introduced above.

Theorem 3. Let $A_{n}(\mathbf{z})=1-\sum_{T \in \mathfrak{P}_{n}^{*}} a_{T} z^{T}, \mathbf{a}=\left(a_{1}, \ldots, a_{n}\right)$, and $Q_{n}$ be the affine polynomial defined in (10) and (11). For any $\alpha$ and $\gamma$ in $\mathbb{N}^{n}$, denote as $c_{\gamma}\left(\lambda, A_{n}\right)$ the coefficient of $\mathbf{z}^{\gamma}$ in the Taylor expansion of $\left[A_{n}(\mathbf{z})\right]^{-\lambda}$ and as $c_{\alpha}\left(\lambda, 1-Q_{n}\right)$ the coefficient of $\mathbf{z}^{\alpha}$ in the Taylor expansion $\left[1-Q_{n}(\mathbf{z})\right]^{-\lambda}$. The following relation can be obtained:

$$
\begin{aligned}
c_{\gamma}\left(\lambda, A_{n}\right)= & \sum_{\alpha+\beta=\gamma} c_{\alpha}\left(\lambda, 1-Q_{n}\right)(\lambda \mathbf{1}+\alpha)_{\beta} \frac{\mathbf{a}^{\beta}}{\beta !} \\
= & \sum_{0 \leqslant \beta_{i} \leqslant \gamma_{i}, i=1, \ldots, n} c_{\gamma-\beta}\left(\lambda, 1-Q_{n}\right) \\
& \times \prod_{i=1}^{n}\left(\lambda+\gamma_{i}-\beta_{i}\right)_{\beta_{i}} \frac{a_{i}^{\beta_{i}}}{\beta_{i} !} \\
= & \sum_{0 \leqslant \alpha_{i} \leqslant \gamma_{i}, i=1, \ldots, n} c_{\alpha}\left(\lambda, 1-Q_{n}\right) \\
& \times \prod_{i=1}^{n}\left(\lambda+\alpha_{i}\right)_{\beta_{i}} \frac{a_{i}^{\gamma_{i}-\alpha_{i}}}{\left(\gamma_{i}-\alpha_{i}\right) !} .
\end{aligned}
$$

The masses of NMDs can be directly obtained from this theorem. The particular cases of bivariate and trivariate NMDs are considered in the following subsections since the corresponding masses will be useful in the application considered in the second part of this paper.

\subsection{Bivariate NMDs}

Theorem 4. Consider the affine polynomial of order 2 with variables $\mathbf{z}=\left(z_{1}, z_{2}\right)$ defined by

$$
A_{2}(\mathbf{z})=1-\sum_{T \in \mathfrak{P}_{2}^{*}} a_{T} z^{T}=1-a_{1} z_{1}-a_{2} z_{2}-a_{1,2} z_{1} z_{2} .
$$

The coefficient of $\mathbf{z}^{\gamma}$ in the Taylor expansion of $\left[A_{2}(\mathbf{z})\right]^{-\lambda}$, can be computed as follows

$$
\begin{aligned}
c_{\gamma}\left(\lambda, P_{2}\right)= & (\lambda)_{\max \left(\gamma_{1}, \gamma_{2}\right)} \sum_{\ell=0}^{\min \left(\gamma_{1}, \gamma_{2}\right)} \frac{(\lambda+\ell)_{\min \left(\gamma_{1}, \gamma_{2}\right)-\ell}}{\left(\gamma_{1}-\ell\right) !\left(\gamma_{2}-\ell\right) ! \ell !} \\
& \times a_{1}^{\gamma_{1}-\ell} a_{2}^{\gamma_{2}-\ell} b_{1,2}^{\ell} .
\end{aligned}
$$

Remark 5. The result (A.11) was mentioned in [7] without the factorization leading to (19). If $a_{1} \neq 0$ and $a_{2} \neq 0$, an equivalent formulation of (19) is

$$
\begin{aligned}
c_{\gamma}\left(\lambda, P_{2}\right)= & \frac{a_{1}^{\gamma_{1}} a_{2}^{\gamma_{2}}}{\gamma_{1} ! \gamma_{2} !}(\lambda)_{\max \left(\gamma_{1}, \gamma_{2}\right)} \\
& \times \sum_{\ell=0}^{\min \left(\gamma_{1}, \gamma_{2}\right)}(\lambda+\ell)_{\min \left(\gamma_{1}, \gamma_{2}\right)-\ell} \\
& \times\left(\begin{array}{c}
\gamma_{1} \\
\ell
\end{array}\right)\left(\begin{array}{c}
\gamma_{2} \\
\ell
\end{array}\right) \ell !\left(\frac{b_{1,2}}{a_{1} a_{2}}\right)^{\ell} .
\end{aligned}
$$

\subsection{Trivariate NMDs}

Theorem 6. Consider the affine polynomial with the three variables $\mathbf{z}=\left(z_{1}, z_{2}, z_{3}\right)$ defined by

$$
A_{3}(\mathbf{z})=1-\sum_{T \in \mathfrak{P}_{3}^{*}} a_{T} z^{T} .
$$


The coefficient of $\mathbf{z}^{\gamma}$ in the Taylor expansion of $\left[A_{3}(\mathbf{z})\right]^{-\lambda}$ are

$$
\begin{aligned}
c_{\gamma}\left(\lambda, P_{3}\right)= & \sum_{\beta_{1}=0}^{\gamma_{1}} \sum_{\beta_{2}=0}^{\gamma_{2}} \sum_{\beta_{3}=0}^{\gamma_{3}} \sum_{v=\|\gamma-\beta\|}^{\lfloor|\gamma-\beta| / 2\rfloor}(\lambda)_{v} \\
& \times \frac{b_{2,3}^{v-\gamma_{1}+\beta_{1}} b_{1,3}^{v-\gamma_{2}+\beta_{2}} b_{1,2}^{v-\gamma_{3}+\beta_{3}}}{\prod_{i=1}^{3}\left(v-\gamma_{i}+\beta_{i}\right) !} \frac{b_{1,2,3}^{|\gamma-\beta|-2 v}}{(|\gamma-\beta|-2 v) !} \\
& \times \frac{\left(\lambda+\gamma_{1}-\beta_{1}\right)_{\beta_{1}}}{\beta_{1} !} \frac{\left(\lambda+\gamma_{2}-\beta_{2}\right)_{\beta_{2}}}{\beta_{2} !} \\
& \times \frac{\left(\lambda+\gamma_{3}-\beta_{3}\right)_{\beta_{3}}}{\beta_{3} !} a_{1}^{\beta_{1}} a_{2}^{\beta_{2}} a_{3}^{\beta_{3}} .
\end{aligned}
$$

When $a_{i} \neq 0, b_{i, j} \neq 0, i \neq j, i=1,2,3, j=1,2,3$, and $b_{1,2,3} \neq 0$, an equivalent expression is

$$
\begin{aligned}
c_{\gamma}\left(\lambda, P_{3}\right)= & a_{1}^{\gamma_{1}} a_{2}^{\gamma_{2}} a_{3}^{\gamma_{3}} \sum_{\beta_{1}=0}^{\gamma_{1}} \sum_{\beta_{2}=0}^{\gamma_{2}} \sum_{\beta_{3}=0}^{\gamma_{3}} \sum_{v=\|\gamma-\beta\|}^{\lfloor|\gamma-\beta| / 2\rfloor}(\lambda)_{v} \\
& \times \frac{\left(b_{2,3} b_{1,3} b_{1,2} / b_{1,2,3}^{2}\right)^{v}}{(|\gamma-\beta|-2 v) ! \prod_{i=1}^{3}\left(v-\gamma_{i}+\beta_{i}\right) !} \\
& \times\left[\prod_{i=1}^{3} \frac{\left.\left(\lambda+\gamma_{i}-\beta_{i}\right)_{\beta_{i}}\right]\left(\frac{b_{1,2,3}}{a_{1} b_{2,3}}\right)^{\gamma_{1}-\beta_{1}}}{\beta_{i} !}\right. \\
& \times\left(\frac{b_{1,2,3}}{a_{2} b_{1,3}}\right)^{\gamma_{2}-\beta_{2}}\left(\frac{b_{1,2,3}}{a_{3} b_{1,2}}\right)^{\gamma_{3}-\beta_{3}} \\
= & (\lambda)_{\|\gamma\|} \frac{\mathbf{a}^{\gamma}}{\gamma !} \sum_{\alpha_{1}=0 \alpha_{2}=0 \alpha_{3}=0}^{\gamma_{1}} \sum_{v=\|\alpha\|}^{\gamma_{3}} \frac{(\lambda|\alpha|-2\rfloor}{(|\alpha|-2 v) ! \prod_{i=1}^{3}\left(v-\alpha_{i}\right) !} \\
& \times\left(\frac{b_{2,3} b_{1,3} b_{1,2}}{b_{1,2,3}^{2}}\right)^{v} \alpha !\left(\frac{b_{1,2,3}}{a_{1} b_{2,3}}\right)^{\alpha_{1}}\left(\frac{b_{1,2,3}}{a_{2} b_{1,3}}\right)^{\alpha_{2}}\left(\frac{b_{1,2,3}}{a_{3} b_{1,2}}\right)^{\alpha_{3}} \\
& \times \prod_{i=1}^{3}\left[\left(\lambda+\alpha_{i}\right)_{\gamma_{i}-\alpha_{i}}\left(\begin{array}{l}
\gamma_{i} \\
\alpha_{i}
\end{array}\right)\right] .
\end{aligned}
$$

\section{Estimating the Polarization Degree of Low Flux Polarimetric Images Using Maximum Likelihood Methods}

4.1. Low Flux Polarimetric Images. The state of the polarization of the light can be described by the random behavior of a complex vector $\mathbf{A}=\left(A_{X}, A_{Y}\right)$, called the Jones vector, whose covariance matrix, called the polarization matrix, is

$$
\Gamma=\left(\begin{array}{ll}
\mathbb{E}\left[A_{X} A_{X}^{*}\right] & \mathbb{E}\left[A_{X} A_{Y}^{*}\right] \\
\mathbb{E}\left[A_{Y} A_{X}^{*}\right] & \mathbb{E}\left[A_{Y} A_{Y}^{*}\right]
\end{array}\right) \triangleq\left(\begin{array}{cc}
a_{1} & a_{3}+i a_{4} \\
a_{3}-i a_{4} & a_{2}
\end{array}\right),
$$

where $*$ denotes the complex conjugate. The covariance matrix $\Gamma$ is a nonnegative Hermitian matrix whose diagonal terms are the intensity components in the $X$ and $Y$ directions. The cross terms of $\Gamma$ are the correlations between the Jones components. If we assume a fully developed speckle, the Jones vector $\mathbf{A}$ is distributed according to a complex Gaussian distribution whose probability density function (pdf) is [15]:

$$
p(\mathbf{A})=\frac{1}{\pi^{2}|\Gamma|} \exp \left(-\mathbf{A}^{\dagger} \Gamma^{-1} \mathbf{A}\right)
$$

where $|\Gamma|$ is the determinant of the matrix $\Gamma$ and $\dagger$ denotes the conjugate transpose operator. As a consequence, the statistical properties of $\mathbf{A}$ are fully characterized by the covariance matrix $\Gamma$. The different components of $\Gamma$ can be classically estimated by using four intensity images that are related to the components of the Jones vector as follows (see [20], for more details):

$$
\begin{aligned}
& I_{1}=\left|A_{X}\right|^{2}, \\
& I_{2}=\left|A_{Y}\right|^{2}, \\
& I_{3}=\frac{1}{2}\left|A_{X}\right|^{2}+\frac{1}{2}\left|A_{Y}\right|^{2}+\operatorname{Re}\left(A_{X} A_{Y}^{*}\right), \\
& I_{4}=\frac{1}{2}\left|A_{X}\right|^{2}+\frac{1}{2}\left|A_{Y}\right|^{2}+\operatorname{Im}\left(A_{X} A_{Y}^{*}\right) .
\end{aligned}
$$

The state of the polarization of the light is classically characterized by the square DoP defined by [15, pages $134-136]$

$$
P^{2}=1-4 \frac{|\Gamma|}{[\operatorname{trace}(\Gamma)]^{2}}=1-\frac{4\left[a_{1} a_{2}-\left(a_{3}^{2}+a_{4}^{2}\right)\right]}{\left(a_{1}+a_{2}\right)^{2}},
$$

where trace $(\Gamma)$ is the trace of the matrix $\Gamma$. The light is totally depolarized for $P=0$, totally polarized for $P=1$, and partially polarized when $P \in] 0,1[$. As a consequence, estimating the square DoP of a polarimetric image is important in many practical applications. Different estimation methods of $P^{2}$ using several combinations of intensity images were studied in [20]. Since only one realization of the random vector $\mathbf{I}=$ $\left(I_{1}, \ldots, I_{4}\right)^{T}$ was available for a given pixel of a polarimetric image, the image was supposed to be locally stationary and ergodic. These assumptions were used to derive square DoP estimators using several neighbor pixels belonging to a socalled estimation window.

This paper considers practical applications where the intensity level of the reflected light is very low (low flux assumption), which leads to an additional source of fluctuations on the detected signal. Under the low flux assumption, the quantum nature of the light leads to a Poisson-distributed noise which can become very important relatively to the mean value of the signal at a low photon level. As a consequence, the observed pixels of the low flux polarimetric image are discrete random variables contained in the vector $\mathbf{N}=$ $\left(N_{1}, \ldots, N_{4}\right)$ such that the conditional distributions of the random variables $N_{l} \mid I_{l}$, for $l=1, \ldots, 4$ are independent and distributed according to Poisson distributions with means $I_{l}$, 
for $l=1, \ldots, 4$. The resulting joint distribution of $\mathbf{N}$ is a multivariate mixed Poisson distribution [22]:

$$
P(\mathbf{N}=\mathbf{k})=\int_{\left(\mathbb{R}^{+}\right)^{4}}^{\ldots} \int \prod_{l=1}^{4} \frac{I_{l}^{k_{l}}}{k_{l} !} \exp \left(-I_{l}\right) f(\mathbf{I}) d \mathbf{I}
$$

where $\mathbf{k}=\left(k_{1}, \ldots, k_{4}\right), k_{i} \in \mathbb{N}$, and $f(\mathbf{I})$ is the joint pdf of the intensity vector. This section studies estimators of the square DoP $P^{2}$ defined in (28) based on several vectors $\mathbf{N}^{1}, \ldots, \mathbf{N}^{n}$ belonging to the estimation window. These estimators are constructed from estimates of the covariance matrix elements $a_{i}, i=1, \ldots, 4$. As explained in the introduction, several studies have been recently devoted to the estimation of the square DoP using less than four polarimetric images. This paper goes into this direction by deriving estimators based on the observation of 2 or 3 polarimetric images.

The joint distribution of the intensity vector $\mathbf{I}$ is known to be a multivariate gamma distribution whose Laplace transform is [20]

$$
E\left[\exp \left(\sum_{k=1}^{4} z_{k} I_{k}\right)\right]=\frac{1}{P_{4}(\mathbf{z})}
$$

where the affine polynomial $P_{4}$ is

$$
P_{4}(\mathbf{z})=1+\mathbf{z} \boldsymbol{\mu}+k_{a}\left[2 z_{1} z_{2}+z_{3} z_{4}+\left(z_{1}+z_{2}\right)\left(z_{3}+z_{4}\right)\right]
$$

with $\mathbf{z}=\left(z_{1}, \ldots, z_{4}\right)$ and

$$
\begin{gathered}
k_{a}=\frac{1}{2}\left(a_{1} a_{2}-a_{3}^{2}-a_{4}^{2}\right) \\
\boldsymbol{\mu}=\left(a_{1}, a_{2}, a_{3}+\frac{a_{1}+a_{2}}{2}, a_{4}+\frac{a_{1}+a_{2}}{2}\right)^{T} .
\end{gathered}
$$

As a consequence, the distribution of $\mathbf{N}$ is an NMD whose PGF can be written as (the interested reader is invited to consult [22] for more details)

$$
G_{\mathbf{N}}(\mathbf{z})=\frac{1}{P_{4}\left(z_{1}-1, z_{2}-1, z_{3}-1, z_{4}-1\right)} .
$$

The results of Section 2 allow us to compute the masses of $\mathbf{N}$ that will be useful for studying the maximum likelihood estimator (MLE) of the square DoP.

4.2. MLE Using Three Polarimetric Images. The PGF of $\widetilde{\mathbf{N}}=$ $\left(N_{1}, N_{2}, N_{3}\right)$ can be computed from (33) by setting $z_{4}=1$. The following result can be obtained:

$$
G_{\widetilde{\mathbf{N}}}(\mathbf{z})=\frac{1}{P_{3}(\mathbf{z})}
$$

with

$$
\begin{aligned}
P_{3}(\mathbf{z})= & P_{3}(\mathbf{0})+z_{1}\left(\mu_{1}-3 k_{a}\right)+z_{2}\left(\mu_{2}-3 k_{a}\right) \\
& +z_{3}\left(\mu_{3}-2 k_{a}\right)+k_{a}\left(2 z_{1} z_{2}+z_{1} z_{3}+z_{2} z_{3}\right),
\end{aligned}
$$

$\mathbf{z}=\left(z_{1}, z_{2}, z_{3}\right)$, and $P_{3}(\mathbf{0})=1-\sum_{i=1}^{3} \mu_{i}+4 k_{a}$. The results of Section 3.2 can then be used to express the masses of $\widetilde{\mathbf{N}}$ as a function of $\boldsymbol{\theta}=\left(a_{1}, a_{2}, a_{3}, a_{4}^{2}\right)^{T}$.

The ML estimator of $\boldsymbol{\theta}$ based on several vectors $\widetilde{\mathbf{N}}^{k}$ belonging to the estimation window (where $k=1, \ldots, K$ and $K$ is the number of pixels of the observation window) is obtained by maximizing the log-likelihood

$$
l_{3}\left(\widetilde{\mathbf{N}}^{1}, \ldots, \widetilde{\mathbf{N}}^{K} \mid \boldsymbol{\theta}\right)=\sum_{k=1}^{K} \log \left[P\left(\widetilde{\mathbf{N}}^{k}\right)\right]
$$

with respect to $\boldsymbol{\theta}$ (note again that $P\left(\widetilde{\mathbf{N}}^{k}\right)$ is the mass of a trivariate NMD that has been computed in Section 3.2). The practical determination of the ML estimator of $\boldsymbol{\theta}$ is achieved by using a Newton-Raphson procedure. The ML estimators of the vector $\boldsymbol{\theta}$, denoted as $\widetilde{\boldsymbol{\theta}}=\left(\widetilde{a}_{1}, \tilde{a}_{2}, \tilde{a}_{3},{\widetilde{a_{4}^{2}}}^{T}\right.$, are then plugged into (28) to provide the ML estimator of the square DoP based on three polarimetric images:

$$
\widetilde{P}^{2}=1-\frac{4\left[\widetilde{a}_{1} \widetilde{a}_{2}-\left(\widetilde{a}_{3}^{2}+\widetilde{a}_{4}^{2}\right)\right]}{\left(\widetilde{a}_{1}+\widetilde{a}_{2}\right)^{2}} .
$$

4.3. MLE Using Two Polarimetric Images. The PGF of $\underline{\mathbf{N}}=$ $\left(N_{1}, N_{2}\right)$ can be computed from (34) by setting $z_{3}=1$. The following result can be obtained:

$$
G_{\underline{\mathbf{N}}}(\mathbf{z})=\frac{1}{P_{2}(\mathbf{z})}
$$

with

$$
P_{2}(\mathbf{z})=P_{2}(\mathbf{0})+z_{1}\left(\mu_{1}-2 k_{a}\right)+z_{2}\left(\mu_{2}-2 k_{a}\right)+2 k_{a} z_{1} z_{2},
$$

$\mathbf{z}=\left(z_{1}, z_{2}\right)$ and $P_{2}(\mathbf{0})=1-\sum_{i=1}^{2} \mu_{i}+2 k_{a}$. The results of Section 3.1 can then be used to express the masses of $\underline{\mathbf{N}}$ as a function of $\boldsymbol{\theta}=\left(a_{1}, a_{2}, k_{a}\right)^{T}$.

The MLE of $\boldsymbol{\theta}$ based on several vectors $\underline{\mathbf{N}}^{k}$ belonging to the estimation window is obtained by maximizing the loglikelihood

$$
l_{2}\left(\underline{\mathbf{N}}^{1}, \ldots, \underline{\mathbf{N}}^{K} \mid \boldsymbol{\theta}\right)=\sum_{k=1}^{K} \log \left[P\left(\underline{\mathbf{N}}^{k}\right)\right]
$$

with respect to $\boldsymbol{\theta}$ (note that $P\left(\underline{\mathbf{N}}^{k}\right)$ is the mass of a bivariate NMD that has been computed in Section 3.1). The practical determination of the ML estimator of $\boldsymbol{\theta}$ is achieved by using a Newton-Raphson procedure. The ML estimators of the vector $\boldsymbol{\theta}$ elements, denoted as $\underline{\boldsymbol{\theta}}=\left(\underline{a}_{1}, \underline{a}_{2}, k_{a}\right)^{T}$, are then plugged into (28) to provide the MLE of the square DoP based on two polarimetric images:

$$
\underline{P}^{2}=1-\frac{8 \underline{k}_{a}}{\left(\underline{a}_{1}+\underline{a}_{2}\right)^{2}} .
$$


TABLE 1: Covariance matrix elements and square DoP values for the Jones vector.

\begin{tabular}{ccccccccccccc}
\hline & $\Gamma_{0}$ & $\Gamma_{1}$ & $\Gamma_{2}$ & $\Gamma_{3}$ & $\Gamma_{4}$ & $\Gamma_{5}$ & $\Gamma_{6}$ & $\Gamma_{7}$ & $\Gamma_{8}$ & $\Gamma_{9}$ & $\Gamma_{10}$ \\
\hline$a_{1}$ & 2.00 & 1.71 & 2.40 & 1.82 & 2.00 & 2.67 & 2.40 & 2.24 & 2.74 & 2.00 \\
$a_{2}$ & 2.00 & 2.29 & 1.60 & 2.18 & 2.00 & 1.33 & 1.60 & 1.76 & 1.26 & 2.00 & 2.00 \\
$a_{3}$ & 0.00 & 0.40 & 0.48 & 0.91 & 0.89 & 0.96 & 1.07 & 1.05 & 1.46 & 0.60 & 1.41 \\
$a_{4}$ & 0.00 & 0.40 & 0.64 & 0.58 & 0.89 & 0.80 & 1.05 & 1.28 & 0.73 & 1.80 & 1.41 \\
\hline$P^{2}$ & 0 & 0.1 & 0.2 & 0.3 & 0.4 & 0.5 & 0.6 & 0.7 & 0.8 & 0.9 & 1.0 \\
\hline
\end{tabular}

TABLE 2: Simulation results for the estimation of $P^{2}$ using 2 or 3 images, obtained from 1000 Monte-Carlo runs $(n=51 \times 51)$.

\begin{tabular}{|c|c|c|c|c|c|c|c|c|}
\hline & \multicolumn{4}{|c|}{2 images MLE } & \multicolumn{4}{|c|}{3 images MLE } \\
\hline & Bias & std & MSE & avar & Bias & std & MSE & avar \\
\hline$\Gamma_{0}$ & $3.57 e-03$ & $1.31 e-02$ & $1.85 e-04$ & - & $4.12 e-03$ & $9.08 e-03$ & $9.93 e-05$ & - \\
\hline$\Gamma_{1}$ & $4.28 e-04$ & $3.14 e-02$ & $9.83 e-04$ & $9.78 e-04$ & $1.71 e-03$ & $2.57 e-02$ & $6.62 e-04$ & $7.25 e-04$ \\
\hline$\Gamma_{2}$ & $9.49 e-04$ & $3.23 e-02$ & $1.04 e-03$ & $9.82 e-04$ & $1.20 e-03$ & $2.86 e-02$ & $8.18 e-04$ & $7.83 e-04$ \\
\hline$\Gamma_{3}$ & $-2.15 e-04$ & $3.13 e-02$ & $9.81 e-04$ & $9.67 e-04$ & $4.84 e-04$ & $2.88 e-02$ & $8.28 e-04$ & $8.07 e-04$ \\
\hline$\Gamma_{4}$ & $2.49 e-03$ & $3.02 e-02$ & $9.19 e-04$ & $8.91 e-04$ & $1.71 e-03$ & $2.81 e-02$ & $7.91 e-04$ & $7.67 e-04$ \\
\hline$\Gamma_{5}$ & $2.86 e-04$ & $2.85 e-02$ & $8.10 e-04$ & $7.54 e-04$ & $4.80 e-04$ & $2.65 e-02$ & $7.03 e-04$ & $6.69 e-04$ \\
\hline$\Gamma_{6}$ & $5.25 e-04$ & $2.59 e-02$ & $6.72 e-04$ & $6.58 e-04$ & $6.32 e-04$ & $2.47 e-02$ & $6.11 e-04$ & $5.95 e-04$ \\
\hline$\Gamma_{7}$ & $1.05 e-03$ & $2.37 e-02$ & $5.60 e-04$ & $5.43 e-04$ & $1.04 e-03$ & $2.26 e-02$ & $5.11 e-04$ & $4.95 e-04$ \\
\hline$\Gamma_{8}$ & $1.59 e-04$ & $2.01 e-02$ & $4.03 e-04$ & $4.12 e-04$ & $3.17 e-04$ & $1.90 e-02$ & $3.60 e-04$ & $3.73 e-04$ \\
\hline$\Gamma_{9}$ & $-2.88 e-04$ & $1.76 e-02$ & $3.10 e-04$ & $3.20 e-04$ & $-4.46 e-04$ & $1.65 e-02$ & $2.73 e-04$ & $2.86 e-04$ \\
\hline$\Gamma_{10}$ & $-5.98 e-03$ & $8.98 e-03$ & $1.16 e-04$ & - & $-5.73 e-03$ & $8.58 e-03$ & $1.06 e-04$ & - \\
\hline
\end{tabular}

\section{Simulation Results}

5.1. Estimation Performance. The performance of the ML estimators of the square DoP based on two or three polarimetric images has been evaluated via several experiments. The first simulations compare the log Mean Square Errors (MSEs) of the square DoP estimators constructed from two or three images. Eleven different covariance matrices of the Jones vector have been considered in order to define typical values of the DoP. The values of $a_{i}$, for $i=1, \ldots, 4$, (defining the covariance matrix elements of the Jones vector) and the corresponding values of the square DoPs are reported in Table 1 . Note that all the covariance matrices $\Gamma_{j}, j=0, \ldots, 10$, have been normalized so that the mean of the total intensity $\mathbf{E}\left[I_{1}+I_{2}\right]=\mathbf{E}\left[N_{1}+N_{2}\right]=a_{1}+a_{2}$ is equal to 4 . Thus, the average number of photons collected on each pixel equals 4 for each matrix of the considered set. This point is in agreement with the low flux assumption.

Figure 1(a) display the empirical log MSEs of the square DoP estimators for the set of covariance matrices defined in Table 1 as a function of the true square DoP value. The red plus markers + correspond to the estimators obtained for two polarimetric images (2D MLE), whereas the blue cross markers $\times$ correspond to the MLE obtained for three polarimetric images ( $3 \mathrm{D} \mathrm{MLE}$ ). Note that these empirical MSEs have been computed for a square observation window of size $n=51 \times 51$ pixels, based on 1000 Monte-Carlo runs. The theoretical asymptotic log MSEs of the MLE are also displayed in Figure 1(a) with continuous lines. These asymptotic values correspond to the Cramer-Rao Lower Bound (CRLB) for the parameter $P^{2}$. The MLE is known to be asymptotically unbiased and efficient under mild regularity conditions (that are satisfied for $P^{2}$ ). Thus, the MSE of the estimates can be approximated a for large sample by the CRLB. More details about the way of computing the square DoP CLRBs can be found in [23]. Figure 1(a) indicates that the empirical MSEs are in good agreement with the corresponding CRLBs, except for the matrices $\Gamma_{0}$ and $\Gamma_{10}$. Indeed, the CRLBs for these two matrices cannot be computed since the true value of the parameters belongs to the boundary of its definition domain. The empirical bias, standard deviations ("std"), MSEs, and asymptotic variances ("avar") of the estimators of $P^{2}$ are also reported in Table 2. It is interesting to note that the MLE obtained using 3 images is slightly more biased than the one obtained using 2 images. However, the MLE based on 3 images provides lower MSEs than the estimator based on 2 images, as expected.

In order to appreciate the influence of the Poisson noise due to the low flux assumption, experiments have been conducted using the high flux assumption. In this case, the intensity vector $I$ is assumed to be known. Thus, the high flux MLEs using two and three images can be derived from the intensity vectors $\underline{\mathbf{I}}^{k}=\left(I_{1}^{k}, I_{2}^{k}\right)^{T}$ and $\widetilde{\mathbf{I}}^{k}=\left(I_{1}^{k}, I_{2}^{k}, I_{3}^{k}\right)^{T}[20]$. The results are depicted in Figure 1(b). A comparison between Figures 1(a) and 1(b) allows one to appreciate a similar global behaviour for all the estimators, with a maximum MSE near $P^{2}=1 / 3$ and decreasing MSEs as $P^{2}$ goes to 0 or 1 . The degradation of the estimation performance due to the presence of Poisson noise (due to the low flux assumption) can also be clearly noticed.

The next set of simulations studies the performance of the different estimators as a function of the sample size $n$. Figures 2(a) and 2(b) show the log MSEs of the square DoP 


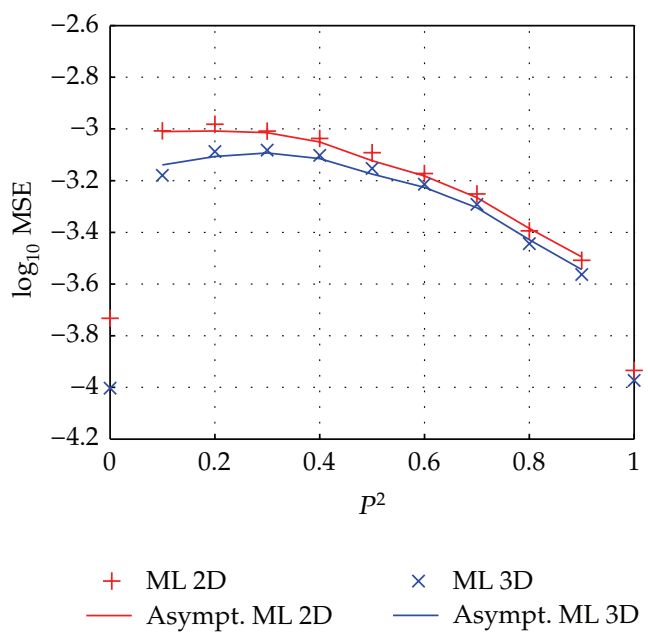

(a) Low flux

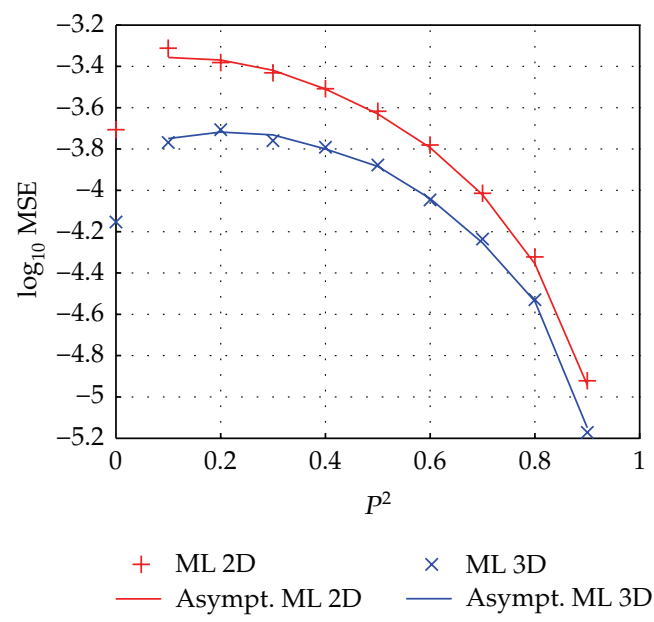

(b) High flux

FIgURE 1: $\log$ MSEs of the square DoP estimates using 2 and 3 images versus $P^{2}$ for the set of polarization matrices defined in Table 1 under (a) low flux and (b) high flux assumptions $(n=51 \times$ 51, ML: maximum likelihood estimators, and Asympt.: theoretical asymptotic value of the log MSE for a given estimator).

estimates obtained for 2 and 3 images (for the two particular matrices $\Gamma_{2}$ and $\Gamma_{8}$ ). The empirical bias, standard deviations ("std"), MSEs, and asymptotic variances ("avar") are also reported in Tables 3 and 4 . One can see that the empirical MSEs are in good agreement with their theoretical asymptotic values for a large enough sample size, that is, $n>25 \times 25$. Moreover, the gain of a performance using 3 images instead of 2 is more significant for small values of $P^{2}$ (indeed, the difference between the different curves is more pronounced in the left figure corresponding to $P^{2}=0.2$ than in the right figure corresponding to $P^{2}=0.8$.)

Figures 3(a) and 3(b) display the MSEs of the MLE under the high flux assumption. By comparing Figures 2 and 3, one can observe that the gain of performance using 3 images instead of 2 is more important in the high flux scenario than under a low flux assumption.

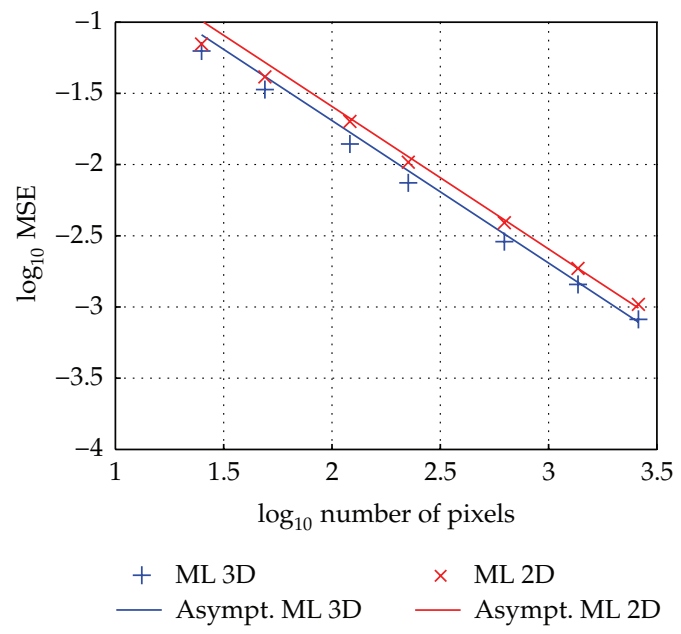

(a) Matrix $\Gamma_{2}$

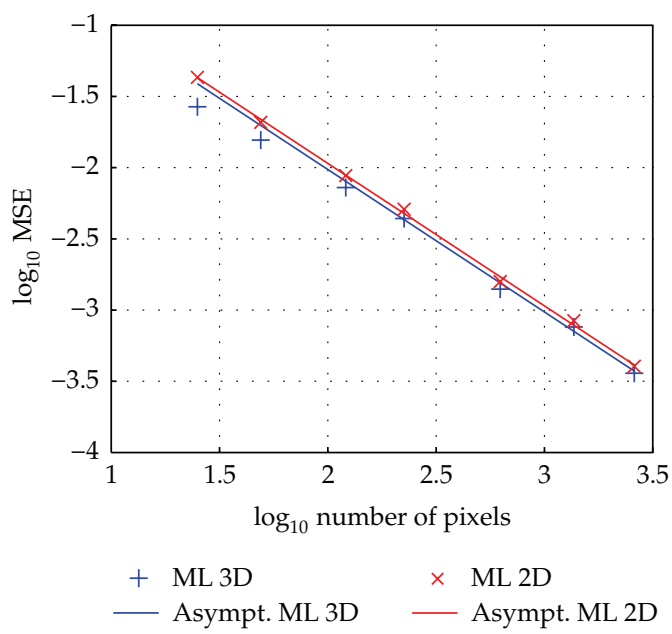

(b) Matrix $\Gamma_{8}$

FIGURE 2: $\log$ MSE of the estimated square DoP $P^{2}$ using 2 or 3 intensity images versus the logarithm of the sample size for the matrices (a) $\Gamma_{2}$ and (b) $\Gamma_{8}$ (ML: maximum likelihood estimators, and Asympt. theoretical asymptotic value of the log MSE for a given estimator).

5.2. Application to Synthetic Polarimetric Images. In order to appreciate the estimation performance on polarimetric images, we consider a synthetic polarimetric image of size $512 \times 512$ composed of three distinct objects located on a homogeneous background depicted in Figure 4 (see also [20]). The polarimetric properties of these objects and background (i.e., the covariance matrix of the Jones vector and the square DoPs) are reported in Table 5. The polarimetric low flux images generated according to this model are also represented in Figure 5 in negative colors (bright pixels correspond to a small number of photons, dark ones correspond to a large number of photons). Note that these images represent the values of the vector $\mathbf{N}=\left(N_{1}, N_{2}, N_{3}, N_{4}\right)^{T}$ for each pixel. The square DoP of each pixel $\mathbf{x}^{(i, j)}$ (for $i, j=1, \ldots, 512$ ) has been estimated from vectors belonging to windows of size $n=15 \times 15$ centered around the pixel of coordinates $(i, j)$ in 


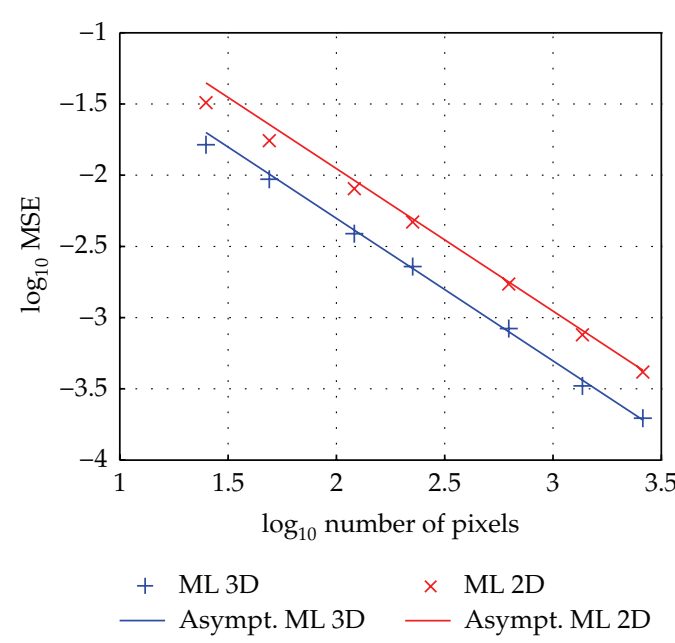

(a) Matrix $\Gamma_{2}$

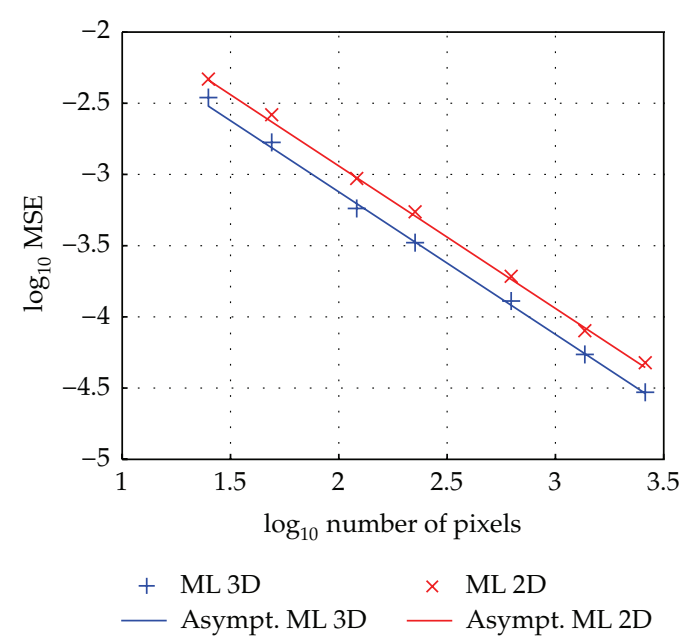

(b) Matrix $\Gamma_{8}$

FIGURE 3: $\log$ MSE of the estimated square DoP $P^{2}$ using 2 or 3 intensity images versus the logarithm of the sample size for the matrices (a) $\Gamma_{2}$ and (b) $\Gamma_{8}$ under high flux assumption (ML: maximum likelihood estimators, Asympt.: theoretical asymptotic value of the log MSE for a given estimator).

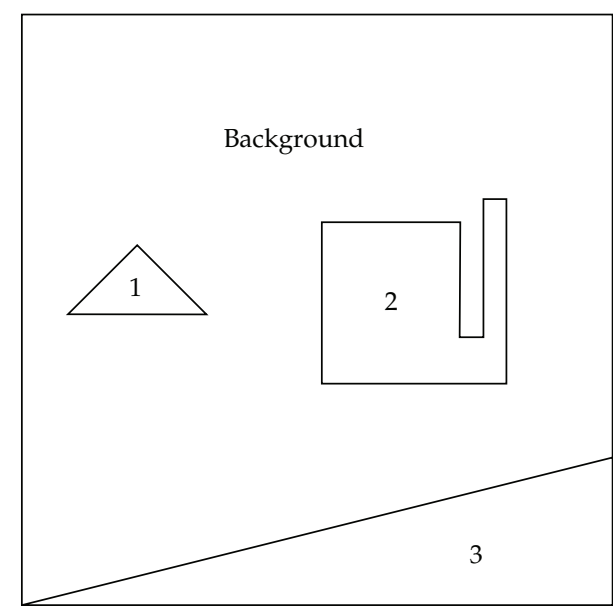

(a) Scene

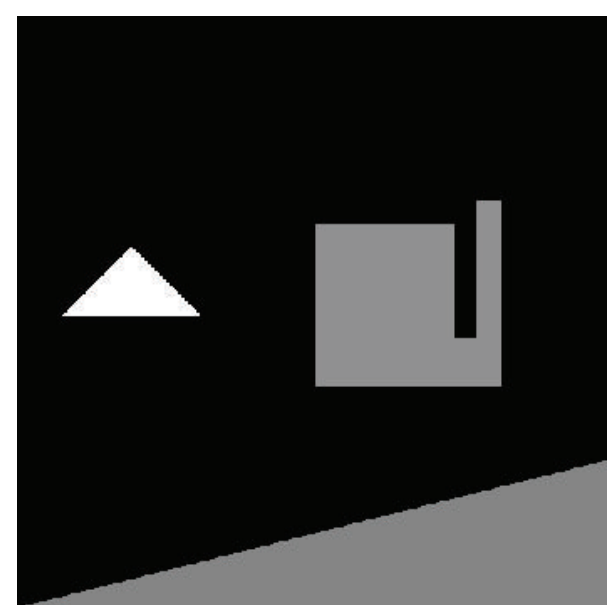

(b) Theoretical squared DoP

FIgURE 4: Composition of the scene used to generate synthetic polarimetric low flux images and associated theoretical squared DoP.

the analyzed image. The estimated square DoPs are depicted in Figures 6(a) and 6(b) for the MLEs using 2 and 3 images, respectively. One can see that the ML method for 3 images gives more homogeneous results on each object than the ML method derived for 2 images. This result confirms that the MLE using 3 images performs better than the MLE using only 2 images. However, the polarimetric properties of a scene can be clearly recovered with 2 or 3 low flux images.

\subsection{Estimation Results on Real-Word Polarimetric Images.} Finally, the ML estimator based on three images is applied on real polarimetric data. These images are acquired by using a laser as a coherent illumination source. The scene consists of two disks. The first one, intended to provide low DoP, is a grey diffuse material (left object in Figure 7). The second one is made of sand blasted aluminium providing high DoP (right object in Figure 7). Due to the experimental conditions, the measured intensities are quite low. As a consequence, these intensities are assumed to be distributed according to an NMD. The intensity images corresponding to $N_{1}, N_{2}, N_{3}$, and $\mathrm{N}_{4}$ are depicted in Figure 7. The interested reader is invited to read [20] for more details on these data. It is important to note that the two disks exhibit the similar level of total reflectivity $N_{1}+N_{2}$ and can hardly be distinguished without a polarimetric processing.

Figure 8 shows the ML estimates of the square DoPs $P^{2}$ for 3 images and an estimation window of size $n=9 \times 9$ pixels. As expected, the values of the estimates are quite different on each disk: quite higher on the metal than on the plastic disk. This result is in good agreement with the theoretical 
TABLE 3: Simulation results for the estimation of $P^{2}$ using 2 or 3 images, obtained from 1000 Monte-Carlo runs (matrix $\Gamma_{2}$ ).

\begin{tabular}{|c|c|c|c|c|c|c|c|c|}
\hline \multirow{2}{*}{$n$} & \multicolumn{4}{|c|}{2 images MLE } & \multicolumn{4}{|c|}{3 images MLE } \\
\hline & Bias & std & MSE & avar & Bias & std & MSE & avar \\
\hline $5^{2}$ & $9.03 e-02$ & $2.49 e-01$ & $7.01 e-02$ & $1.02 e-01$ & $1.20 e-01$ & $2.20 e-01$ & $6.27 e-02$ & $8.15 e-02$ \\
\hline $7^{2}$ & $5.10 e-02$ & $1.97 e-01$ & $4.13 e-02$ & $5.21 e-02$ & $7.04 e-02$ & $1.69 e-01$ & $3.36 e-02$ & $4.16 e-02$ \\
\hline $11^{2}$ & $2.60 e-02$ & $1.39 e-01$ & $2.01 e-02$ & $2.11 e-02$ & $3.22 e-02$ & $1.14 e-01$ & $1.39 e-02$ & $1.68 e-02$ \\
\hline $15^{2}$ & $7.68 e-03$ & $1.02 e-01$ & $1.04 e-02$ & $1.14 e-02$ & $1.59 e-02$ & $8.48 e-02$ & $7.43 e-03$ & $9.05 e-03$ \\
\hline $25^{2}$ & $-7.42 e-04$ & $6.25 e-02$ & $3.90 e-03$ & $4.09 e-03$ & $2.16 e-03$ & $5.36 e-02$ & $2.87 e-03$ & $3.26 e-03$ \\
\hline $37^{2}$ & $-5.19 e-04$ & $4.32 e-02$ & $1.87 e-03$ & $1.87 e-03$ & $6.87 e-05$ & $3.79 e-02$ & $1.44 e-03$ & $1.49 e-03$ \\
\hline $51^{2}$ & $9.49 e-04$ & $3.23 e-02$ & $1.04 e-03$ & $9.82 e-04$ & $1.20 e-03$ & $2.86 e-02$ & $8.18 e-04$ & $7.83 e-04$ \\
\hline
\end{tabular}

TABLE 4: Simulation results for the estimation of $P^{2}$ using 2 or 3 images, obtained from 1000 Monte-Carlo runs (matrix $\Gamma_{8}$ ).

\begin{tabular}{|c|c|c|c|c|c|c|c|c|}
\hline \multirow{2}{*}{$n$} & \multicolumn{4}{|c|}{2 images MLE } & \multicolumn{4}{|c|}{3 images MLE } \\
\hline & Bias & std & MSE & avar & Bias & std & MSE & avar \\
\hline $5^{2}$ & $-3.91 e-03$ & $2.08 e-01$ & $4.31 e-02$ & $4.29 e-02$ & $2.80 e-02$ & $1.61 e-01$ & $2.67 e-02$ & $3.88 e-02$ \\
\hline $7^{2}$ & $4.04 e-03$ & $1.44 e-01$ & $2.08 e-02$ & $2.19 e-02$ & $1.89 e-02$ & $1.24 e-01$ & $1.56 e-02$ & $1.98 e-02$ \\
\hline $11^{2}$ & $2.14 e-03$ & $9.38 e-02$ & $8.80 e-03$ & $8.86 e-03$ & $8.70 e-03$ & $8.47 e-02$ & $7.24 e-03$ & $8.01 e-03$ \\
\hline $15^{2}$ & $-1.27 e-03$ & $7.14 e-02$ & $5.10 e-03$ & $4.76 e-03$ & $2.70 e-03$ & $6.63 e-02$ & $4.39 e-03$ & $4.31 e-03$ \\
\hline $25^{2}$ & $5.23 e-04$ & $3.98 e-02$ & $1.58 e-03$ & $1.71 e-03$ & $6.87 e-04$ & $3.75 e-02$ & $1.40 e-03$ & $1.55 e-03$ \\
\hline $37^{2}$ & $2.24 e-03$ & $2.89 e-02$ & $8.42 e-04$ & $7.83 e-04$ & $2.54 e-03$ & $2.75 e-02$ & $7.61 e-04$ & $7.08 e-04$ \\
\hline $51^{2}$ & $1.59 e-04$ & $2.01 e-02$ & $4.03 e-04$ & $4.12 e-04$ & $3.17 e-04$ & $1.90 e-02$ & $3.60 e-04$ & $3.73 e-04$ \\
\hline
\end{tabular}

properties of the considered material. This emphasizes the interest in considering efficient estimators based on NMDs for polarimetric images.

\section{Appendix}

\section{Proofs of the Theorems}

Proof of Theorem 1. The set of affine polynomials with real coefficients and variables $\left(z_{1}, \ldots, z_{n}\right)$ is a vector space of dimension $2^{n}$ spanned by the basis $\left(\mathbf{z}^{T}\right)_{T \in \mathfrak{P}_{n}}$. The set of polynomials $\left(\mathbf{z}^{T} \prod_{t \in[n] \backslash T}\left(1-a_{t} z_{t}\right)\right)_{T \in \mathfrak{P}_{n}}$ is another basis of this vector space. The proof of the theorem is obtained by expressing the coefficients of $A_{n}$ in this latter basis. Considering the expansion

$$
\begin{aligned}
A_{n}\left(z_{1}, \ldots, z_{n}\right)= & d_{\emptyset}^{n} \prod_{i \in[n]}\left(1-a_{i} z_{i}\right) \\
& -\sum_{T \in \mathfrak{P}_{n}^{*}} d_{T}^{n} \mathbf{z}^{T} \prod_{i \in[n] \backslash T}\left(1-a_{i} z_{i}\right),
\end{aligned}
$$

the following results can be obtained.

(1) Substituting $\mathbf{z}=\mathbf{0}$ in (A.1) leads to $d_{\emptyset}^{n}=1$.

(2) Substituting $\mathbf{z}_{i}=\left(\delta_{i}(1), \ldots, \delta_{i}(n)\right)$ in (A.1), where $\delta_{i}(j)=1$ if $i=j$ and $\delta_{i}(j)=0$ else leads to

$$
1-a_{i}=1-a_{i}-d_{\{i\}}^{n},
$$

or equivalently

$$
d_{\{i\}}^{n}=0, \quad i \in[n] .
$$

(3) Without loss of generality, if $|T|=k>1$, the coefficients $d_{T}^{n}$ can be computed for $T=\{1, \ldots, k\}$. Indeed, consider a permutation $\sigma$ such that $T=$ $\{\sigma(1), \ldots, \sigma(k)\}$. If $d_{\{1, \ldots, k\}}^{n}=f\left(\left\{a_{S}\right\}_{S \in \mathfrak{P}_{k}^{*}}\right)$, we have $d_{T}^{n}=f\left(\left\{a_{\sigma(S)}\right\}_{S \in \mathfrak{P}_{k}^{*}}\right)$. Using the relation

$$
\begin{gathered}
1-A_{n}\left(z_{1}, \ldots, z_{n-1}, 0\right)=\sum_{T \in \mathfrak{P}_{n-1}^{*}} a_{T} z^{T} \\
=1-A_{n-1}\left(z_{1}, \ldots, z_{n-1}\right),
\end{gathered}
$$

the expansion

$$
\begin{aligned}
A_{n}\left(z_{1}, \ldots, z_{n}\right)= & \prod_{i \in[n]}\left(1-a_{i} z_{i}\right) \\
& -\sum_{T \in \mathfrak{P}_{n}^{*},|T| \geqslant 2} d_{T}^{n} \mathbf{z}^{T} \prod_{i \in[n] \backslash T}\left(1-a_{i} z_{i}\right),
\end{aligned}
$$

yields for any $k<n$,

$$
d_{[k]}^{n}=d_{[k]}^{n-1} .
$$

In order to determine $d_{[n]}^{n}$, we can assume $a_{i} \neq 0, i \in$ $[n]$ and substitute $z_{i}=1 / a_{i}, i \in[n]$ in (A.5). The following result is obtained

$$
1-n-\sum_{T \in \mathfrak{P}_{n}^{*},|T| \geqslant 2} \frac{a_{T}}{a^{T}}=-\frac{d_{T}^{n}}{a^{[n]}},
$$


TABLE 5: Polarimetric properties of elements that compose the scene displayed in Figure 4.

\begin{tabular}{|c|c|c|c|}
\hline Object & Polarization matrix $\Gamma$ & $P^{2}$ & Remarks \\
\hline Background & $\left(\begin{array}{cc}0.79 & 0 \\
0 & 0.98\end{array}\right)$ & 0.0115 & Very depolarizing and dark background \\
\hline 1 & $\left(\begin{array}{cc}3.6 & 0 \\
0 & 0.22\end{array}\right)$ & 0.783 & Very bright and weakly depolarizing object (typically steel) \\
\hline 2 & $\left(\begin{array}{cc}3 & 0.1 \\
0.1 & 0.6\end{array}\right)$ & 0.447 & Bright object quite depolarizing \\
\hline 3 & $\left(\begin{array}{cc}0.7 & 0.5+0.2 i \\
0.5-0.2 i & 1.07\end{array}\right)$ & 0.414 & $\begin{array}{c}\text { Dark object whose mean total intensity is the same as the } \\
\text { background }\end{array}$ \\
\hline
\end{tabular}

(a) Low flux intensity $N_{1}$

(c) Low flux intensity $N_{3}$ (b) Low flux intensity $\mathrm{N}_{2}$

(d) Low flux intensity $\mathrm{N}_{4}$

FIgURE 5: Synthetic intensity images (negative colors) for the scene depicted in Figure 4 and described in Table 5.

hence

$$
\begin{aligned}
d_{[n]}^{n} & =\left[(n-1)+\sum_{T \in \mathfrak{P}_{n}^{*},|T| \geqslant 2} \frac{a_{T}}{a^{T}}\right] a^{[n]} \\
& =\sum_{T \in \mathfrak{P}_{n}^{*},|T| \geqslant 2} a_{T} a^{[n] \backslash T}+(n-1) a^{[n]} .
\end{aligned}
$$

Proof of Theorem 3. The relation (10) leads to

$$
\begin{aligned}
{\left[A_{n}(\mathbf{z})\right]^{-\lambda}=} & \left(1-Q_{n}\left(\frac{z_{1}}{1-a_{1} z_{1}}, \ldots, \frac{z_{n}}{1-a_{n} z_{n}}\right)\right)^{-\lambda} \\
& \times\left[\prod_{i=1}^{n}\left(1-a_{i} z_{i}\right)\right]^{-\lambda} \\
= & \left(\sum_{\alpha \in \mathbb{N}^{n}} c_{\alpha}\left(\lambda, 1-Q_{n}\right)\left(\frac{\mathbf{z}}{\mathbf{1 - \mathbf { a z }}}\right)^{\alpha}\right)
\end{aligned}
$$




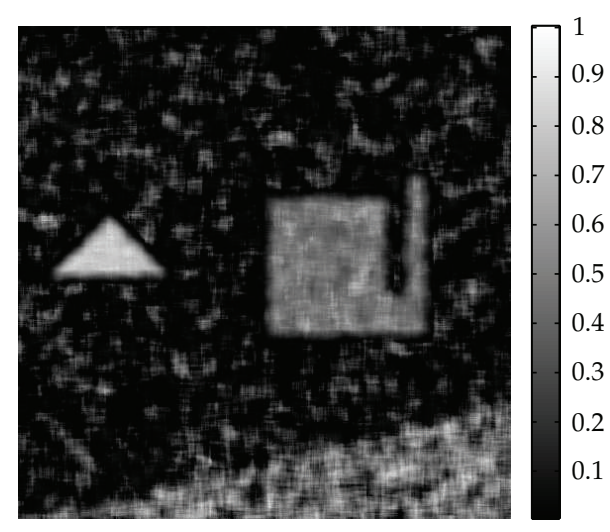

(a) MLE 2 images

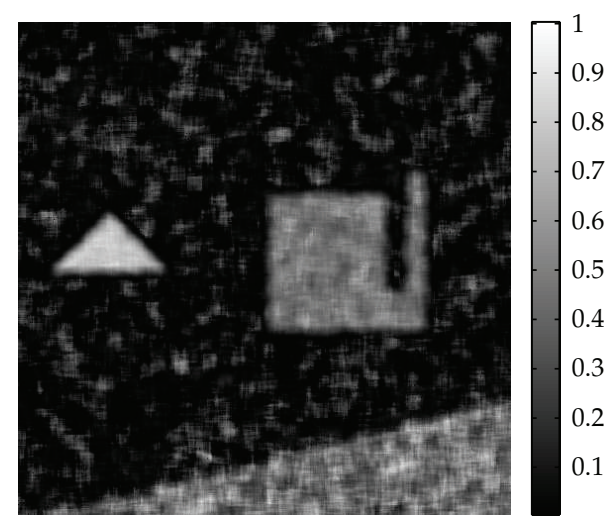

(b) MLE 3 images

FIgURE 6: Estimates of $P^{2}$ using 2 or 3 low flux intensity images for the synthetic polarimetric images for an estimation window of size $n=15 \times 15$. MLE: maximum likelihood estimator.

$$
\begin{aligned}
& \times\left[\prod_{i=1}^{n}\left(1-a_{i} z_{i}\right)\right]^{-\lambda} \\
= & \left(\sum_{\alpha \in \mathbb{N}^{n}} c_{\alpha}\left(\lambda, 1-Q_{n}\right) \mathbf{z}^{\alpha}(\mathbf{1}-\mathbf{a z})^{-(\lambda \mathbf{1}+\alpha)}\right) \\
= & \sum_{\alpha \in \mathbb{N}^{n}} c_{\alpha}\left(\lambda, 1-Q_{n}\right) \mathbf{z}^{\alpha}\left(\sum_{\beta \in \mathbb{N}^{n}}(\lambda \mathbf{1}+\alpha)_{\beta} \frac{\mathbf{a}^{\beta} \mathbf{z}^{\beta}}{\beta !}\right) \\
= & \sum_{\alpha \in \mathbb{N}^{n} \beta \in \mathbb{N}^{n}} c_{\alpha}\left(\lambda, 1-Q_{n}\right)\left((\lambda \mathbf{1}+\alpha)_{\beta} \frac{\mathbf{a}^{\beta}}{\beta !}\right) \mathbf{z}^{\alpha+\beta} \\
= & \sum_{\gamma \in \mathbb{N}^{n}}\left(\sum_{\alpha+\beta=\gamma} c_{\alpha}\left(\lambda, 1-Q_{n}\right)(\lambda \mathbf{1}+\alpha)_{\beta} \frac{\mathbf{a}^{\beta}}{\beta !}\right) \mathbf{z}^{\gamma}
\end{aligned}
$$

which proves (15). Straightforward computations allow us to obtain the equalities (16) and (17) from (15).

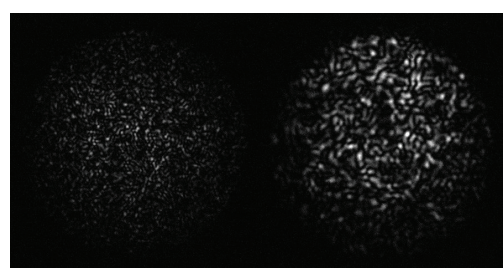

(a) Low flux intensity $N_{1}$

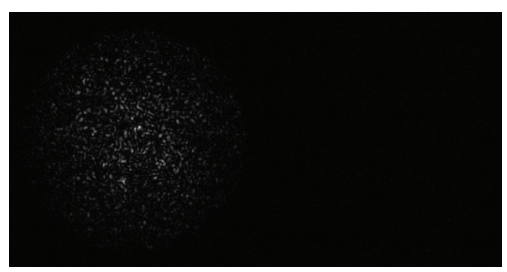

(b) Low flux intensity $\mathrm{N}_{2}$

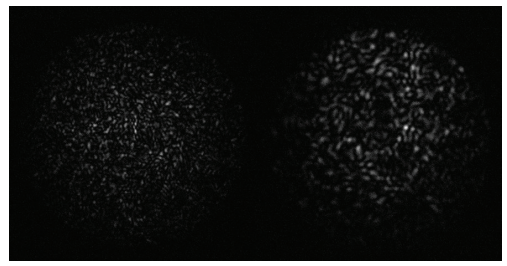

(c) Low flux intensity $\mathrm{N}_{3}$

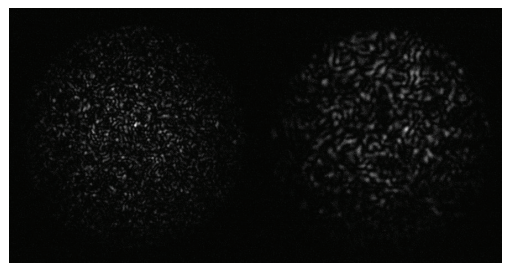

(d) Low flux intensity $\mathrm{N}_{4}$

FIGURE 7: Real-world polarimetric intensity images of a scene composed of a plastic disk (left) and a steel disk (right).

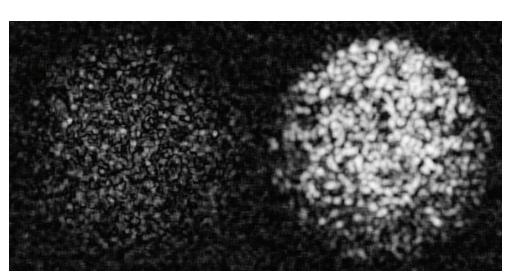

FIgURE 8: Estimates of $P^{2}$ using the 3 low flux intensity images $N_{1}, N_{2}$, and $N_{3}$ for the real polarimetric images for an estimation window of size $n=9 \times 9$. "MLE": maximum likelihood estimator.

Proof of Theorem 4. Denote $\|\alpha\|=\max _{i=1, \ldots, n}\left(\alpha_{i}\right),|\alpha|=$ $\sum_{i=1}^{n} \alpha_{i}$ and introduce the notation of [6]

$$
c_{\alpha}\left(\lambda, P_{n}\right)=\sum_{k \in K_{\alpha}}(\lambda)_{|k|} \frac{\mathbf{a}^{k}}{k !},
$$


where $(\lambda)_{k}=\lambda(\lambda+1) \cdots(\lambda+k-1)=\Gamma(\lambda+k) / \Gamma(\lambda)$. By using Theorem 3 , the following results can be obtained

$$
\begin{aligned}
& c_{\gamma}\left(\lambda, P_{2}\right)=\sum_{\alpha+\beta=\gamma} c_{\alpha}\left(\lambda, 1-Q_{2}\right)(\lambda \mathbf{1}+\alpha)_{\beta} \frac{\mathbf{a}^{\beta}}{\beta !} \\
& =\sum_{\ell=0}^{\min \left(\gamma_{1}, \gamma_{2}\right)}(\lambda)_{\ell}(\lambda+\ell)_{\gamma_{1}-\ell}(\lambda+\ell)_{\gamma_{2}-\ell} \\
& \times \frac{a_{1}^{\gamma_{1}-\ell} a_{2}^{\gamma_{2}-\ell} b_{1,2}^{\ell}}{\left(\gamma_{1}-\ell\right) !\left(\gamma_{2}-\ell\right) ! \ell !} \\
& =\sum_{\ell=0}^{\min \left(\gamma_{1}, \gamma_{2}\right)} \frac{\Gamma(\lambda+\ell)}{\Gamma(\lambda)} \frac{\Gamma\left(\lambda+\gamma_{1}\right)}{\Gamma(\lambda+\ell)} \frac{\Gamma\left(\lambda+\gamma_{2}\right)}{\Gamma(\lambda+\ell)} \\
& \times \frac{a_{1}^{\gamma_{1}-\ell} a_{2}^{\gamma_{2}-\ell} b_{1,2}^{\ell}}{\left(\gamma_{1}-\ell\right) !\left(\gamma_{2}-\ell\right) ! \ell !} \\
& =\frac{\Gamma\left(\lambda+\max \left(\gamma_{1}, \gamma_{2}\right)\right)}{\Gamma(\lambda)} \sum_{\ell=0}^{\min \left(\gamma_{1}, \gamma_{2}\right)} \frac{\Gamma\left(\lambda+\min \left(\gamma_{1}, \gamma_{2}\right)\right)}{\Gamma(\lambda)} \\
& \times \frac{\Gamma(\lambda)}{\Gamma(\lambda+\ell)} \frac{b_{1,2}^{\ell}}{l !}\left(\prod_{i=1}^{2} \frac{a_{i}^{\gamma_{i}-\ell}}{\left(\gamma_{i}-\ell\right) !}\right) \\
& =(\lambda)_{\max \left(\gamma_{1}, \gamma_{2}\right)} \sum_{\ell=0}^{\min \left(\gamma_{1}, \gamma_{2}\right)} \frac{(\lambda+\ell)_{\min \left(\gamma_{1}, \gamma_{2}\right)-\ell}}{\left(\gamma_{1}-\ell\right) !\left(\gamma_{2}-\ell\right) ! \ell !} \\
& \times a_{1}^{\gamma_{1}-\ell} a_{2}^{\gamma_{2}-\ell} b_{1,2}^{\ell} \text {. }
\end{aligned}
$$

Proof of Theorem 6. The relation (16) with $Q_{3}(\mathbf{z})=b_{1,2} z_{1} z_{2}+$ $b_{1,3} z_{1} z_{3}+b_{2,3} z_{2} z_{3}+b_{1,2,3} z_{1} z_{2} z_{3}$ leads to (22). By using the trivial equality

$$
\begin{gathered}
b_{2,3}^{v-\gamma_{1}+\beta_{1}} b_{1,3}^{v-\gamma_{2}+\beta_{2}} b_{1,2}^{v-\gamma_{3}+\beta_{3}} b_{1,2,3}^{|\gamma-\beta|-2 v} a_{1}^{\beta_{1}} a_{2}^{\beta_{2}} a_{3}^{\beta_{3}} \\
=\left(\frac{b_{2,3} b_{1,3} b_{1,2}}{b_{1,2,3}^{2}}\right)^{v}\left(\prod_{i=1}^{3} a_{i}^{\gamma_{i}}\right)\left(\frac{b_{1,2,3}}{a_{1} b_{2,3}}\right)^{\gamma_{1}-\beta_{1}} \\
\quad \times\left(\frac{b_{1,2,3}}{a_{2} b_{1,3}}\right)^{\gamma_{2}-\beta_{2}}\left(\frac{b_{1,2,3}}{a_{3} b_{1,2}}\right)^{\gamma_{3}-\beta_{3}}
\end{gathered}
$$

we easily obtain (23). Note that for $\|\alpha\| \leqslant v \leqslant\lfloor|\alpha| / 2\rfloor$, we have $(\lambda)_{v}=(\lambda)_{\|\alpha\|}(\lambda+\|\alpha\|)_{v-\|\alpha\|}$. By substituting $\alpha_{i}=\gamma_{i}-\beta_{i}$, $i=1,2,3$ in (23), the last result (24) can be obtained.

\section{Acknowledgments}

The authors would like to thank Gérard Letac for fruitful discussions regarding multivariate gamma distributions and negative multinomial distributions. The Authors are also grateful to Mehdi Alouini for acquiring and providing them the polarimetric images.

\section{References}

[1] D. C. Doss, "Definition and characterization of multivariate negative binomial distribution," Journal of Multivariate Analysis, vol. 9, no. 3, pp. 460-464, 1979.

[2] N. L. Johnson, S. Kotz, and N. Balakrishnan, Discrete Multivariate Distributions, John Wiley \& Sons, New York, NY, USA, 1997.

[3] F. Le Gall, "The modes of a negative multinomial distribution," Statistics and Probability Letters, vol. 76, no. 6, pp. 619-624, 2006.

[4] R. C. Griffiths and R. K. Milne, "A class of infinitely divisible multivariate negative binomial distributions," Journal of Multivariate Analysis, vol. 22, no. 1, pp. 13-23, 1987.

[5] S. K. Bar-Lev, D. Bshouty, P. Enis, G. Letac, I. L. Lu, and D. Richards, "The diagnonal multivariate natural exponential families and their classification," Journal of Theoretical Probability, vol. 7, no. 4, pp. 883-929, 1994.

[6] P. Bernardoff, "Which negative multinomial distributions are infinitely divisible?" Bernoulli, vol. 9, no. 5, pp. 877-893, 2003.

[7] F. Chatelain, S. Lambert-Lacroix, and J. Y. Tourneret, "Pairwise likelihood estimation for multivariate mixed Poisson models generated by gamma intensities," Statistics and Computing, vol. 19, no. 3, pp. 283-301, 2009.

[8] C. Brosseau, Fundamentals of Polarized Light-A Statistical Approach, John Wiley \& Sons, New York, NY, USA, 1998.

[9] J. S. Lee and E. Pottier, Polarimetric Radar Imaging: From Basics to Applications, Optical Science and Engineering, Taylor \& Francis, London, UK, 2009.

[10] S. Huard, Polarization of LightStatistical Optics, John Wiley \& Sons, New York, NY, USA, 1997.

[11] H. Mott, Remote Sensing With Polarimetric Radar, John Wiley \& Sons, New York, NY, USA, 2007.

[12] R. Shirvani, M. Chabert, and J. Y. Tourneret, "Ship and oilspill detection using the degree of polarization in linear and hybrid/compact dual-pol SAR," IEEE Journal of Selected Topics in Applied Earth Observations and Remote Sensing, vol. 5, no. 3, pp. 885-892, 2012.

[13] R. C. Jones, "A new calculus for the treatment of optical systems V. A more general formulation and description of another calculus," vol. 37, no. 2, pp. 107-110, 1947.

[14] E. Wolf, "Coherence properties of partially polarized electromagnetic radiation," Il Nuovo Cimento, vol. 13, no. 6, pp. 11651181, 1959.

[15] J. Goodman, Statistical Optics, John Wiley \& Sons, New York, NY, USA, 1985.

[16] J. Fade, M. Roche, and P. Réfrégier, "Precision of moment-based estimation of the degree of polarization in coherent imagery without polarization device," Journal of the Optical Society of America A, vol. 25, no. 2, pp. 483-492, 2008.

[17] J. Fade, P. Réfrégier, and M. Roche, "Estimation of the degree of polarization from a single speckle intensity image with photon noise," Journal of Optics A, vol. 10, no. 11, Article ID 115301, 2008.

[18] P. Réfrégier, M. Roche, and F. Goudail, "Cramer-Rao lower bound for the estimation of the degree of polarization in active coherent imagery at low photon levels," Optics Letters, vol. 31, no. 24, pp. 3565-3567, 2006.

[19] M. Roche, J. Fade, and P. Réfrégier, "Parametric estimation of the square degree of polarization from two intensity images degraded by fully developed speckle noise," Journal of the Optical Society of America A, vol. 24, no. 9, pp. 2719-2727, 2007. 
[20] F. Chatelain, J. Y. Tourneret, M. Roche, and M. Alouini, "Estimating the polarization degree of polarimetric images in coherent illumination using maximum likelihood methods," Journal of the Optical Society of America A, vol. 26, no. 6, pp. 1348-1359, 2009.

[21] W. B. Sparks and D. J. Axon, "Panoramic polarimetry data analysis," Publications of the Astronomical Society of the Pacific, vol. 111, no. 764, pp. 1298-1315, 1999.

[22] A. Ferrari, G. Letac, and J. Y. Tourneret, "Multivariate mixed poisson distributions," in Proceedings of the EUSIPCO-04, F. Hlawatsch, G. Matz, M. Rupp, and B. Wistawel, Eds., pp. 10671070, Vienna, Austria, September 2004.

[23] F. Chatelain, Lois statistiques multivariees pour le traitement de l'image [Ph.D. thesis], Institut National Polytechnique de Toulouse, Toulouse, France, 2007. 


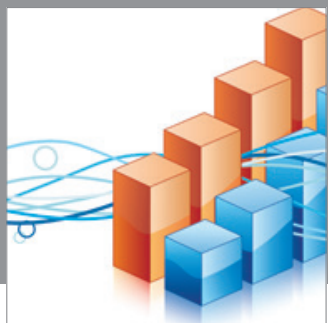

Advances in

Operations Research

mansans

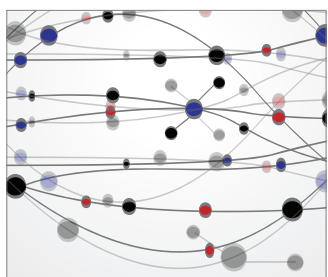

The Scientific World Journal
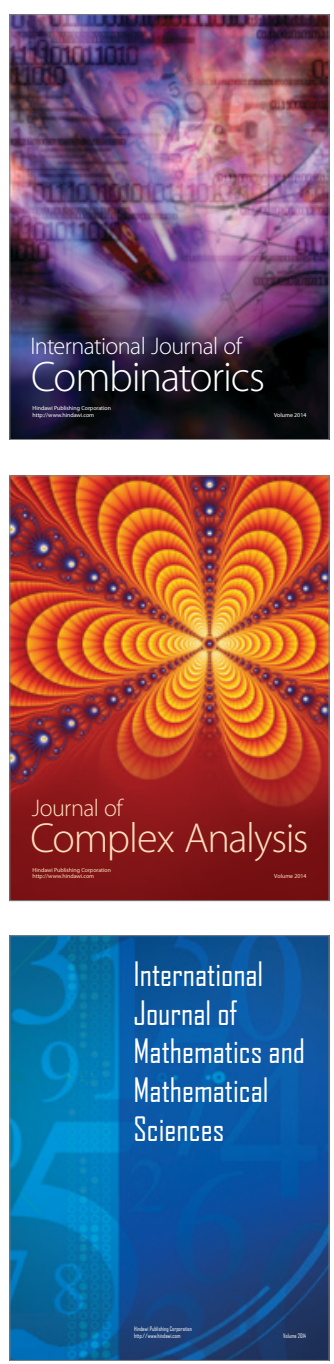
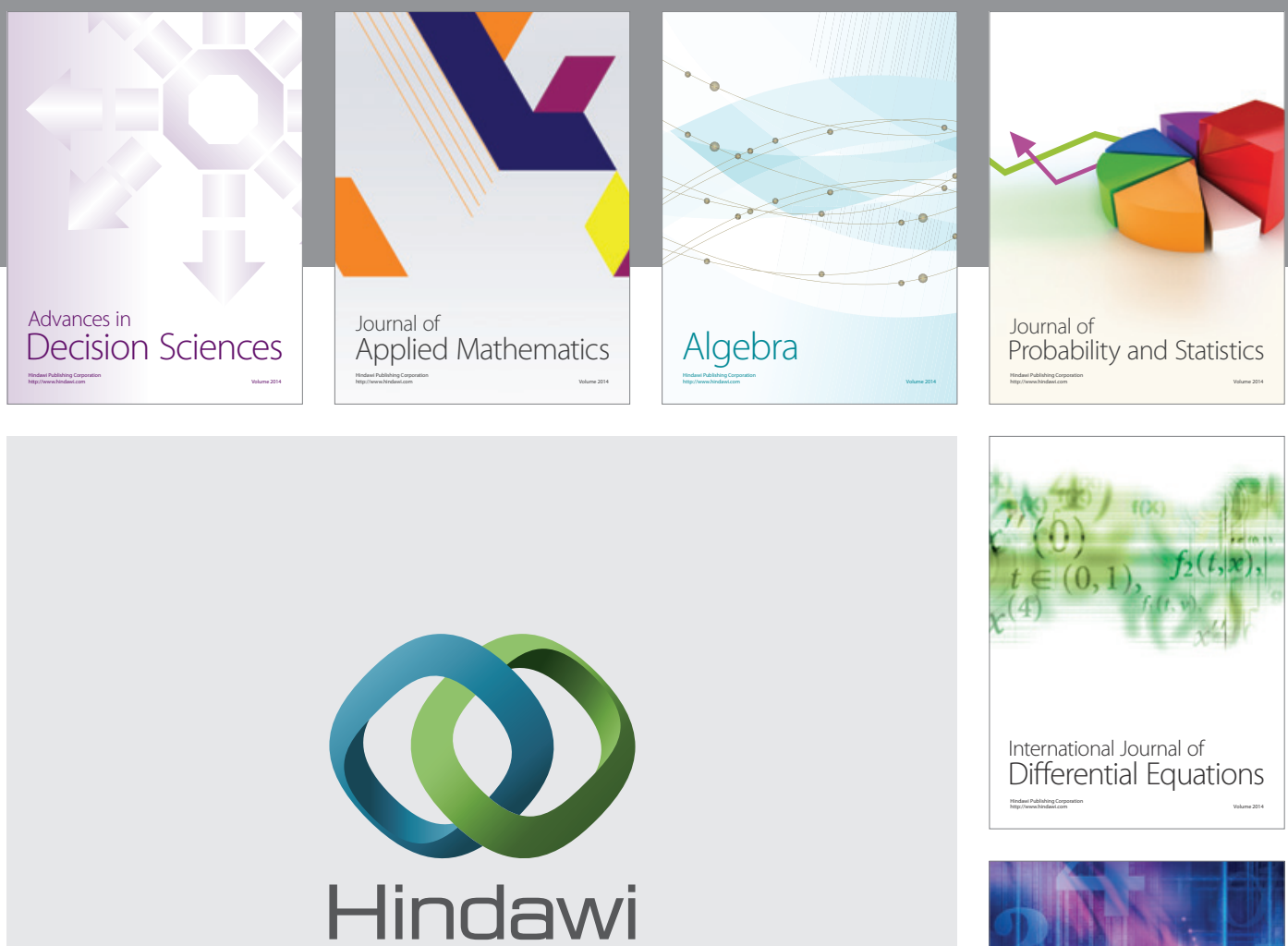

Submit your manuscripts at http://www.hindawi.com
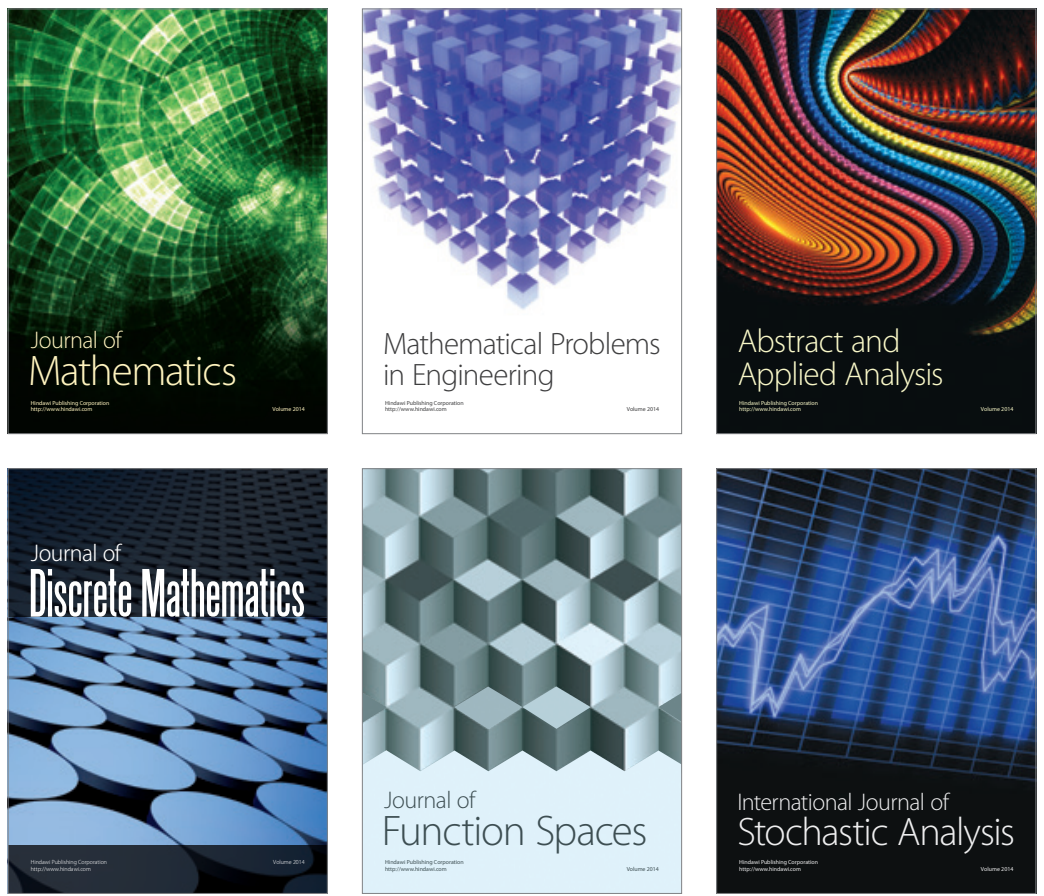

Journal of

Function Spaces

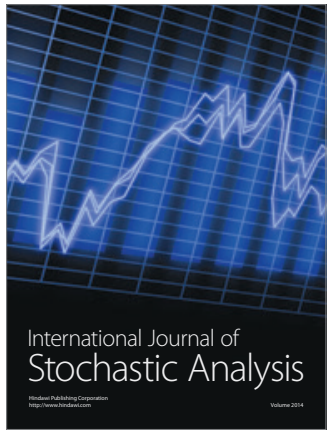

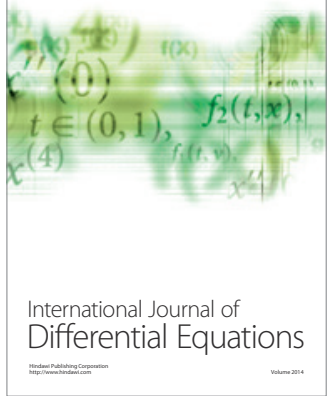
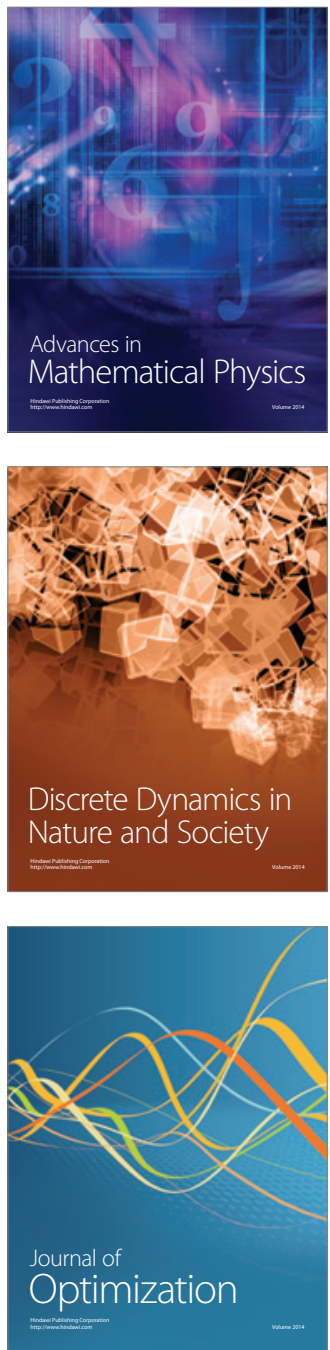